\title{
Anatomical and Functional Connectivity of Cytoarchitectonic Areas within the Human Parietal Operculum
}

\author{
Simon B. Eickhoff, ${ }^{1,2,3}$ Saad Jbabdi, ${ }^{4}$ Svenja Caspers, ${ }^{2,3}$ Angela R. Laird, ${ }^{5}$ Peter T. Fox, ${ }^{5}$ Karl Zilles, $, 3,6$ \\ and Timothy E. J. Behrens ${ }^{4,7}$ \\ ${ }^{1}$ Department of Psychiatry and Psychotherapy, Rheinisch-Westfälische Technische Hochschule Aachen University, D-52074 Aachen, Germany, \\ ${ }^{2}$ Institute of Neuroscience and Medicine (INM-2), Research Centre Jülich, D-52425 Jülich, Germany, ${ }^{3}$ Jülich Aachen Research Alliance, \\ Translational Brain Medicine, D-52425 Jülich, Germany, ${ }^{4}$ Oxford Centre for Functional MRI of the Brain, University of Oxford, John Radcliffe \\ Hospital, Oxford OX3 9D2, United Kingdom, ${ }^{5}$ Research Imaging Center, University of Texas Health Science Institute, San Antonio, Texas 78229, ${ }^{6} \mathrm{C}$. \\ \& 0. Vogt Institute for Brain Research, University of Düsseldorf, D-40225 Düsseldorf, Germany, and 7Department of Experimental Psychology, \\ University of Oxford, Oxford OX1 3UD, United Kingdom
}

In monkeys, the somatosensory cortex on the parietal operculum can be differentiated into several distinct cortical fields. Potential human homologues for these areas have already been defined by cytoarchitectonic mapping and functional imaging experiments. Differences between the two most widely studied areas [operculum parietale (OP) 1 and OP 4] within this region particularly pertain to their connection with either the perceptive parietal network or the frontal motor areas. In the present study, we investigated differences in anatomical connection patterns probed by probabilistic tractography on diffusion tensor imaging data. Functional connectivity was then mapped by coordinate-based meta-analysis of imaging studies. Comparison between these two aspects of connectivity showed a good congruency and hence converging evidence for an involvement of these areas in matching brain networks. There were, however, also several instances in which anatomical and functional connectivity diverged, underlining the independence of these measures and the need for multimodal characterization of brain connectivity. The connectivity analyses performed showed that the two largest areas within the human parietal operculum region display considerable differences in their connectivity to frontoparietal brain regions. In particular, relative to OP 1, area OP 4 is more closely integrated with areas responsible for basic sensorimotor processing and action control, while OP 1 is more closely connected to the parietal networks for higher order somatosensory processing. These results are largely congruent with data on nonhuman primates. Differences between anatomical and functional connectivity as well as between species, however, highlight the need for an integrative view on connectivity, including comparison and cross-validation of results from different approaches.

\section{Introduction}

The primate secondary somatosensory cortex is located on the parietal operculum (Kaas and Collins, 2003). Investigations in many species have provided converging evidence that it can be subdivided into several areas, featuring separate somatotopic maps differing from each other in cytoarchitecture and myeloarchitecture, response properties, and connectivity (Burton et al., 1995; Huffman et al., 1999; Krubitzer and Kaas, 1990; Krubitzer et al., 1995). The area immediately ventral to the anterior parietal somatosensory cortex [areas $3 \mathrm{a}, 3 \mathrm{~b}$, and 1 on the postcentral gyrus (PCG)] is termed the "parietal ventral area" (PV). It is followed caudally by area S2, located on the posterior part of the

Received Nov. 16, 2009; revised March 21, 2010; accepted March 26, 2010.

We acknowledge funding by the Human Brain Project/Neuroinformatics Research (National Institute of Biomedical Imaging and Bioengineering, National Institute of Neurological Disorders and Stroke, National Institute of Mental Health; to K.Z.), the Human Brain Project (R01-MH074457-01A1; to S.B.E.) and the Helmholz Initiative on Systems-Biology "The Human Brain Model" (to K.Z. and S.B.E.).

Correspondence should be addressed to Simon B. Eickhoff at the second address above. E-mail: S.Eickhoff@fz-juelich.de.

DOI:10.1523/JNEUROSCI.5664-09.2010

Copyright $\odot 2010$ the authors $\quad 0270-6474 / 10 / 306409-13 \$ 15.00 / 0$ parietal operculum ventrally to the inferior parietal cortex (IPC). Together, PV and S2 are the most well characterized areas on the parietal operculum. While the term "SII region" is still widely prevalent in the functional neuroimaging literature, the lack of differentiation between the reference to the entire region and a particular area (now denoted S2 to clear up the confusion between SII region and "area SII") may cause unnecessary confusion when comparing findings from different experiments (Eickhoff, 2006c; Burton et al., 2008a, 2008b). We hence opted to avoid this term in the current study in favor of the purely anatomical description "parietal operculum," which comprises areas $\mathrm{S} 2, \mathrm{PV}$, and the "ventral somatosensory area" (VS). The latter is located more medial than areas S2 and PV, shows poorer responsiveness to sensory stimuli, and crude somatotopic organization (Cusick et al., 1989; Krubitzer and Calford, 1992; Qi et al., 2002). More recently, it has been proposed that the latter region may actually consist of two separate rostral and caudal areas, VSr and VSc, respectively (Coq et al., 2004).

A homologous subdivision of the human parietal operculum was initially proposed based on a functional magnetic resonance imaging (fMRI) study providing evidence that the human pari- 
etal operculum may contain several somatotopically organized areas (Disbrow et al., 2000). Subsequently, a postmortem investigation of the parietal operculum revealed the existence of four distinct cytoarchitectonic areas in this region (Eickhoff et al., 2006a, 2006d), which were termed OP (operculum parietale) 1-4. Based on topography and somatotopic organization (Eickhoff et al., 2006c, 2007), OP 4 corresponds to primate area PV, while more caudally OP 1 constitutes the putative human homologue of area S2, and OP 3 is the most likely candidate for macaque area VS. OP 2 is not a somatosensory cortical field but is the homologue of the vestibular region PIVC (parietoinsular vestibular cortex) in nonhuman primates (Eickhoff et al., 2006e).

The functional roles of these human cortical regions are yet a matter of conjecture, as both S2 (OP 4) and PV (OP 1) are coactivated by a wide range of paradigms (Kell et al., 2005; Burton et al., 2008b; Jung et al., 2009). Tracer studies in nonhuman primates, however, have provided some evidence for differences in the connectivity pattern of these two areas (Qi et al., 2002; Disbrow et al., 2003; Kaas and Collins, 2003). Area S2 is regarded as a somatosensory "perceptive" area strongly interconnected with the inferior parietal cortex (Disbrow et al., 2003). In contrast, PV may sustain sensory-motor integration and, at least in new world monkeys such as marmosets, has denser connections with frontal motor and premotor cortices than S2 (Qi et al., 2002). Based on these observations, we here investigated the anatomical and functional connectivity of human areas OP 4 and OP 1.

\section{Materials and Methods}

We investigated the frontoparietal connectivity of human areas OP 4 (S2) and OP 1 (PV) by using two independent approaches. Differences between OP 4 (S2) and OP 1 (PV) with respect to their anatomical connectivity to other frontoparietal regions were probed by probabilistic tractography on diffusion tensor imaging (DTI) data. Differences between OP 4 (S2) and OP 1 (PV) with respect to their functional connectivity with other frontoparietal regions were probed by coordinate-based metaanalysis of imaging studies.

\section{Anatomical connectivity analysis by} probabilistic tractography

Data acquisition. Diffusion-weighted images were acquired in 17 healthy subjects (6 women and 11 men; mean \pm SD age, $26.18 \pm 4.81$; range, 20-38) on a $1.5 \mathrm{~T}$ Siemens Sonata magnetic resonance scanner (maximum gradient strength of $40 \mathrm{mT} / \mathrm{m}$ ). All subjects gave informed written consent into this study, which was approved by the Oxford Research Ethics Committee (Oxford, UK). Diffusion-weighted acquisitions were performed using echo planar imof the parietal operculum.
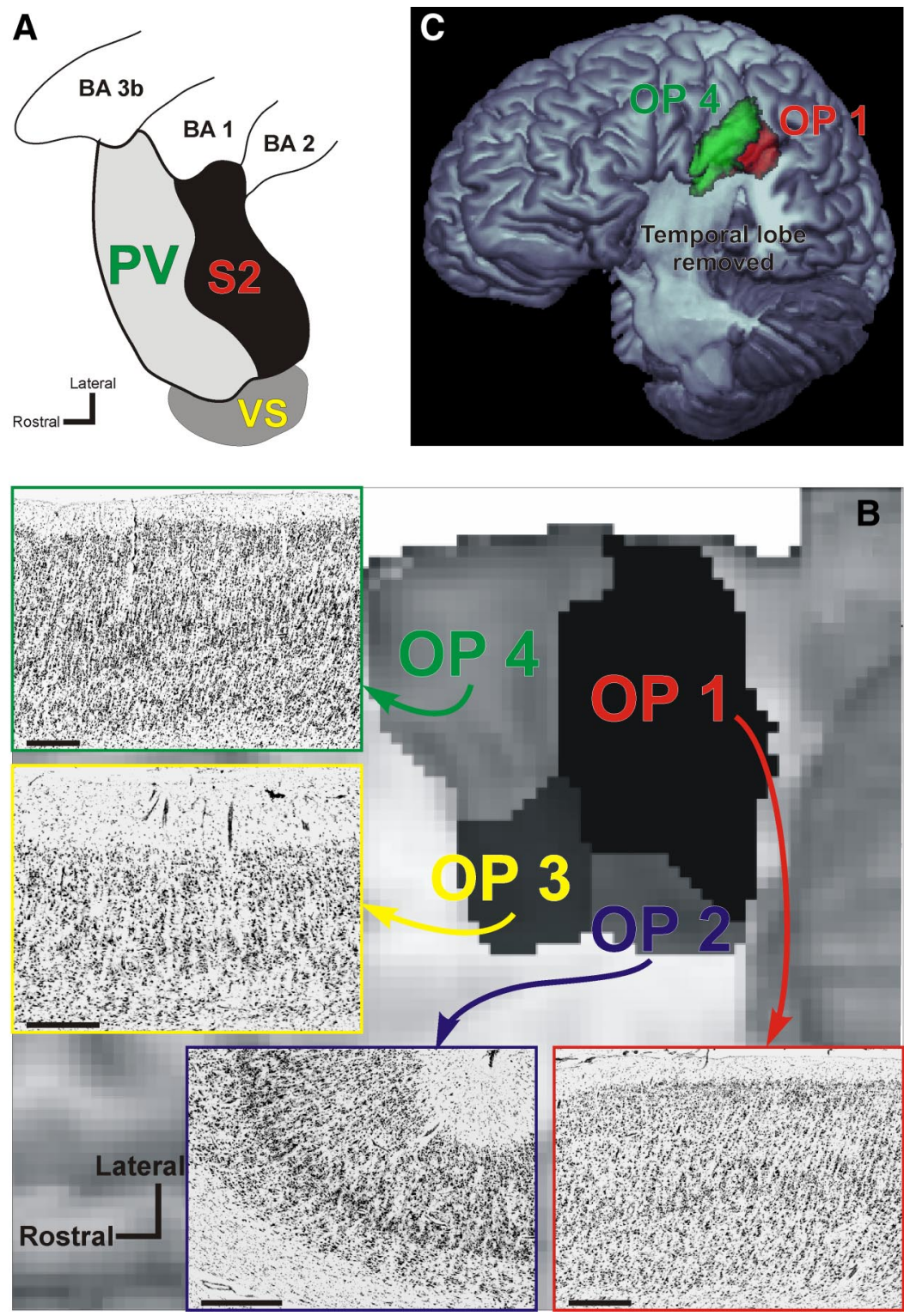

Figure 1. A, Organization of cortical areas in the lateral sulcus of nonhuman primates [adopted and summarized from Disbrow et al. (2003) and Krubitzer et al. (1995)]. It should be noted that inconsistent evidence for further somatosensory areas in this region has been discussed and that a subdivision of VS into two separate areas, VSr (rostral) and VSC (caudal), has been proposed (Coq et al., 2004). B, Anatomical organization of the human parietal operculum. Four distinct cytoarchitectonic areas (termed OP $1-4)$ have been delineated in this region using quantitative histological analysis. Following 3D reconstruction of the cytoarchitectonically analyzed postmortem brains and spatial normalization of these areas into the reference space of the MNI, probabilistic maps for the areas were computed and subsequently combined into an MPM. This anatomical MPM indicates the most likely area at each voxel of the reference space and is shown here in a ventral view on a surface rendering of the MNI single-subject template. The temporal lobes have been removed to obtain an unobstructed view onto the parietal operculum, where the different colors correspond to OP 1-4 as marked. Based on topography and somatotopic organization, OP 4 should correspond to primate area PV, OP 1 to area S2, and OP 3 to area VS. Finally, OP 2 is the homologue of the parietoinsular vestibular cortex in nonhuman primates. Bars in the histological images denote $1 \mathrm{~mm}$. C, Three-dimensional surface rendering of the MNI single-subject template after removal of the temporal lobe. The position of areas OP 1 and OP 4 as defined by their MPM representation is indicated on this view in red and green, respectively. In contrast to panel $\boldsymbol{B}$, which gives a view directly facing the parietal operculum, the tilted view used in this panel provides an overview on the extent of OP 1 and OP 4 on the free surface. Together with $\boldsymbol{B}$, it can be seen that OP 4 encroaches the free surface of the subcentral gyrus while covering about two-thirds of the mediolateral width of the parietal operculum. Area OP 1, on the other hand, barely reaches the free surface but covers about three-fourths of the mediolateral width

aging (voxel size: $2 \times 2 \times 2 \mathrm{~mm}, 60$ isotropically distributed directions; $b$ value: $1000 \mathrm{~s} / \mathrm{mm}^{2}$ ). For each subject, three sets of diffusion-weighted data were obtained. Each set also contained five additional volumes with no diffusion weighting that were acquired distributed throughout the acquisition sequence. 

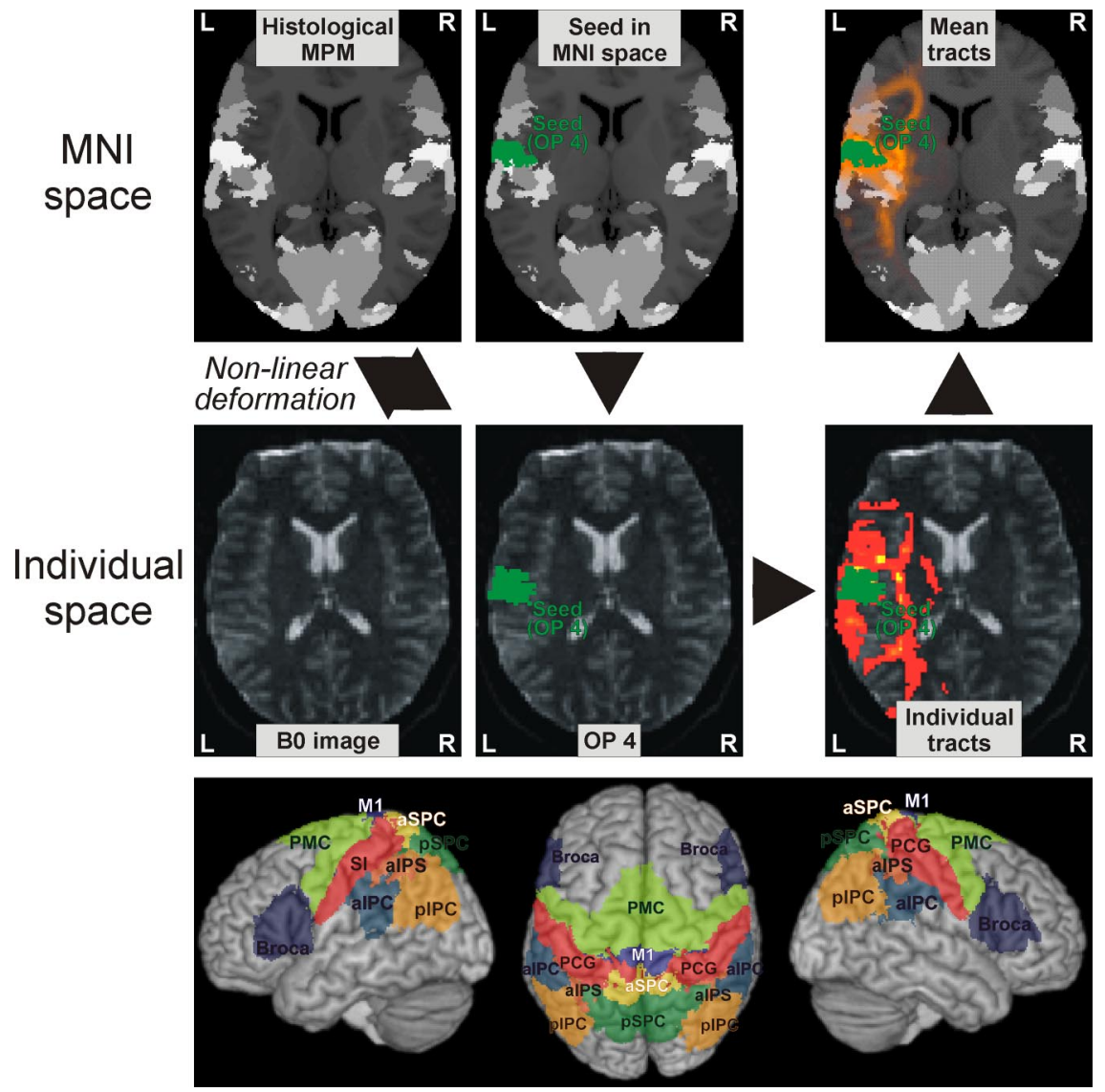

Figure 2. Top and middle panels, Seeds and targets were defined by representations of the respective cytoarchitectonic areas in an MPM of all histologically defined regions. This MPM is shown in the background of the figures in the top row. Different shades of gray denote the different cytoarchitectonic areas. For the sake of display clarity, however, these areas are not individually labeled in these figures. In the displayed example, the seed region was defined by the anatomical location of area $\mathrm{OP} 4$, which is indicated in green. The volume of interest defining this seed, i.e., the location of area $\mathrm{OP} 4$, is then transformed into single-subject diffusion space for probabilistic tractography. Quantitative analysis was then based on the sample count obtained from these analyses, i.e., the number of probabilistic traces originating from the seed (OP 1 or OP 4) reaching a particular target (cf. Table 1). For visualization, the ensuing tracts were also back-projected into the MNI space and averaged to represent the mean pathways. In the figures on the top and middle right, the color scale from red to yellow indicates the probability that the respective tract passes through the respective voxel as obtained from probabilistic tractography. MNI space refers to the reference space defined by the Montreal Neurological Institute for the definition of stereotaxic coordinates (Collins et al., 1994; Evans et al., 1992), which currently represents the most widely used standard for multisubject analysis of neuroimaging data. Bottom panel, Surface rendering of the target regions covering the frontal and parietal cortices displayed on a surface rendering of the MNI template. Each of these cytoarchitectonically defined targets is transformed into the single-subject diffusion space in the same manner as illustrated above for a seed region. An overview on the acronyms used to label the different regions as well as further information on these and their cytoarchitectonic correlates is given in Table 1.

Analysis of diffusion-weighted images was performed using the Functional MRI of the Brain Software Library (FSL) (www.fmrib.ox. ac.uk/fsl). First, all images were realigned to each other by affine registration to compensate for head scan movement and corrected for eddy currents (Smith et al., 2004). The data from the three acquisitions were subsequently averaged to improve the signal-to-noise ratio. We then calculated probability distributions on multiple fiber directions at each voxel in the diffusion data using a multiple fiber extension (Behrens et al., 2007) of a previously described diffusion modeling approach (Behrens et al., 2003a, 2003b). Here, the algorithm was limited to estimating two fiber orientations at each voxel based on the $b$ value and number of gradient orientations in the diffusion data (Rushworth et al., 2006; Tomassini et al., 2007). That is, based on the diffusion-weighted images obtained from applying 60 gradient directions, the probability distributions of up to two independent fiber orientations were estimated at each voxel.

Seed region definition. Seed regions on the parietal operculum were defined by the anatomical locations of cytoarchitectonic areas OP 1 and
OP 4, which are illustrated in Figure $1 B$. The borders of these areas were delineated by microscopic investigation and quantitative histological analysis in a sample of 10 human postmortem brains (Schleicher et al., 2005). These brains were subsequently reconstructed in three dimensions (3D) from digitized images of the histological sections, block-face images taken during the cutting of the brains, and T1 MRI scans obtained before histological processing. These individual brains were subsequently normalized into the reference space defined by the templates (Evans et al., 1992; Collins et al., 1994) provided by the Montreal Neurological Institute (MNI; Montreal, Canada), termed the MNI space. As the next step, a probabilistic cytoarchitectonic map was computed for each area, describing at every voxel of the reference space how likely this area was found at a particular position (Zilles et al., 2002). To generate a discrete parcellation of the cerebral cortex based on the probabilistic information contained in these (overlapping) maps, a summary map [maximum probability map (MPM)] was computed. This map identifies the most likely anatomical area at each voxel of the MNI single-subject template and hence provides a continuous, nonoverlapping map of the human brain (Eickhoff et al., 2005, 2006b). The MPM representations of areas OP 1 and OP 4 were then used as seeds for probabilistic tractography. That is, the regions of interest for which connectivity measures were computed correspond to the anatomical locations of OP 1 and OP 4 as defined by objective cytoarchitectonic analysis following normalization into a standard reference space.

Since, however, these definitions are only available in MNI space, whereas diffusion image analysis is performed in single-subject space, these MPM representations first had to be spatially transformed to match the individual subject's anatomy (Fig. 2). This mapping was achieved by computing transformation fields normalizing each subject's mean $b_{0}$ image and the MNI single-subject template, respectively, to the MNI tissue probability maps (TPMs) using a segmentation-based approach (Ashburner and Friston, 2005). For each subject, the transformation pointing from the MNI single-subject template to the TPMs was then combined with the inverse of the transformation from an individual $b_{0}$ image to the TPMs (mapping from the TPMs to the individual diffusion space). Hereby, the MPM-based seed regions could be transformed from the MNI single-subject template into the diffusion space of each individual subject despite the different image contrasts. Following probabilistic tractography (cf. below), the ensuing tracts were then back-projected into the MNI single-subject space using the same approach and averaged for visualization of white matter pathways connecting OP 1 and OP 4 with frontoparietal targets.

Probabilistic tractography. For each subject, probabilistic tractography was run from the seeds defined by the representations of areas OP 1 and OP 4 in the individual diffusion spaces, using the estimates of (multiple) fiber orientations in each voxel (Behrens et al., 2007). The approach draws a sample from each fiber orientation distribution at the current voxel and chooses the sample closest to the orientation of its previous step. The connection probability between a seed and another voxel in the brain is given by the number of traces arriving at the target site. The aim of this study was to elucidate differential connection patterns of areas 
Table 1. Target regions used for the analysis of anatomical connectivity of areas OP1 and OP4

\begin{tabular}{|c|c|c|c|}
\hline Target region & Acronym & Cytoarchitectonic areas & References \\
\hline \multicolumn{4}{|l|}{ Frontal } \\
\hline Broca's region & Broca & Areas 44,45 & Amunts et al., 1999, 2004 \\
\hline Premotor cortex & PMC & Area 6 & Geyer, 2003 \\
\hline Primary motor cortex & M1 & Areas $4 a, 4 p$ & Geyer et al., 1996 \\
\hline \multicolumn{4}{|l|}{ Parietal } \\
\hline Postcentral gyrus & $P C G$ & Areas $3 a, 3 b, 1,2$ & Geyer et al., 1999, 2000; Grefkes et al., 2001 \\
\hline Anterior intraparietal sulcus & alPS & Areas hIP1, hIP2, hIP3 & Choi et al., 2006; Scheperjans et al., 2008a, 2008b \\
\hline Anterior superior parietal cortex & aSPC & Areas $5 C i, 5 \mathrm{~L}, 5 \mathrm{M}$ & Scheperjans et al., 2008a, 2008b \\
\hline Posterior superior parietal cortex & pSPC & Areas $7 A, 7 M, 7 P, 7 P C$ & Scheperjans et al., 2008a, 2008b \\
\hline Anterior inferior parietal cortex & alPC & Areas PFt, PF, PFm & Caspers et al., 2006, 2008 \\
\hline Posterior inferior parietal cortex & $\mathrm{pIPC}$ & Areas PGa, PGp & Caspers et al., 2006, 2008 \\
\hline \multicolumn{4}{|l|}{ Thalamus } \\
\hline Nuclei preferentially connecting to the somatosensory cortex & VPL/VPI & Ventroposterior lateral and inferior nuclei & Behrens et al., 2003a \\
\hline Nuclei preferentially connecting to the premotor or primary motor cortex & VL/VA & Ventrolateral nuclei, ventral anterior nuclei & Behrens et al., 2003a \\
\hline
\end{tabular}

OP 1 and OP 4. However, many of the strongest differences in connections expected from the macaque monkey (e.g., those toward Broca's region and the inferior parietal lobule) might also distinguish these regions solely in terms of their physical distance from their respective seeds. To ensure that our results could not be caused by any potential bias in tractography toward nearby connections, we corrected probability counts by the length of the pathway. This approach upweighted longer connections (Tomassini et al., 2007) and thus penalized short ones to exclude the possibility that physical distance alone could account for our results.

To evaluate differences in frontoparietal connectivity patterns of OP 1 and OP 4, target regions (outlined in Table 1 and illustrated in the bottom panel of Fig. 2) were created from the histological data using the same approach as that described for OP 1/OP 4 . These targets cover the entire parietal lobe as well as the frontal motor and premotor areas, including Broca's region.

For each subject, we drew 5000 samples from the connectivity distribution (starting from the seed voxels in OP 1/OP 4) and computed the mean probability of connection for each seed-target combination. These values were then normalized on an individual basis by dividing by the total connection probability of each seed and then rescaled by multiplying with the mean total connection probability across all seeds and targets. Finally, connection densities were divided by the size of the target volumes of interest (VOIs), computed on an individual subject basis, and again rescaled by the mean size of all targets. These steps adjusted our data for the size of the seed and target regions.

Statistical analysis on these connection probabilities was performed using MATLAB (MathWorks). Repeated-measures ANOVAs of connection probability values were used to test for influence of within-subject factors of "opercular seed" (OP 1, OP 4), "target" (cf. Table 1), and "hemisphere" (left, right), as well as the interactions thereof.

The main effect of the factor "opercular seed" assessed whether there was a statistically significant difference between OP 1 and OP 4 with respect to the mean connection strength to all targets. The main effect of the factor "target" tested whether the 12 target regions differed from each other with respect to their anatomical connectivity with the parietal operculum (mean connectivity to OP 1 and OP 4). Finally, the interaction between "opercular seed" and "target" tested for differences in the frontoparietal connectivity of OP 1 and OP 4, i.e., whether the distribution of connection strengths to individual targets was different between OP 1 and OP 4. In this context, it is important to consider that in a two-way ANOVA the individual tests are not conditioned on each other; that is, the significance of, e.g., a particular main effect does not depend on the significance or insignificance of the other main effect or the interaction.

The level of significance was $p<0.05$. If the effect of a factor or an interaction was significant, we used a subsequent pairwise multiple comparison procedure to isolate the levels of this factor that differed significantly from each other $(p<0.05$, corrected for multiple comparisons using Tukey's method) (Tukey, 1994).
Functional connectivity analysis by meta-analysis

Functional connectivity analysis was performed by a meta-analysis of published functional imaging results. The concept behind mapping functional connectivity via meta-analysis originates from the notion that functional connectivity should represent the correlation of spatially removed neurophysiologic events, which implies that functionally connected regions should coactivate above chance in functional imaging studies.

This concept of meta-analytic connectivity modeling (MACM) was first used to investigate functional connectivity based on the frequency distributions of concurrent activation foci (Koski and Paus, 2000). Following the emergence of databases on functional neuroimaging results (Fox and Lancaster, 2002; Laird et al., 2009a), this approach was extended to provide voxelwise co-occurrence maps across the whole brain (Toro et al., 2008). The concept of MACM has then been integrated with the activation likelihood estimation (ALE) approach for quantitative metaanalysis (Turkeltaub et al., 2002) to yield functional connectivity maps of the human amygdala (Robinson et al., 2009). More recently, finally, the mapping of functional connectivity via coordinate-based meta-analysis has been validated by comparison to resting-state connectivity (Smith et al., 2009), showing very good concordance between both approaches.

Here, MACM was performed using the BrainMap database (www. brainmap.org), which contains a summary of the results for (at the time of analysis) $\sim 6500$ individual functional neuroimaging experiments. Given the high standardization of neuroimaging data reports and in particular the ubiquitous adherence to standard coordinate systems, the results reported in these studies can readily be compared to each other with respect to the location of significant activation. Using this broad pool of neuroimaging results, MACM can then be used to test for associations between activation probabilities of different areas. Importantly, this inference is performed independently of the applied paradigms or other experimental factors, but rather is solely based on the likelihood of observing activation in a target region [e.g., the premotor cortex (PMC)], given that activation is present within the seed area (e.g., OP 1 or OP 4). Results from such an analysis are therefore robust across many different experimental designs. Database-aided MACM that assesses the coactivation pattern of OP 1 and OP 4 as defined by their MPM representation across a large number of imaging studies should hence allow the delineation and comparison of their functional connectivity. However, functional connectivity per se only allows the delineation of interacting networks but not the causal influences therein. In practice, MACM was performed using the following approach. Studies causing activation within OP 1 or OP 4 were obtained through the BrainMap database. Criteria for retrieval were as follows: only fMRI and positron emission tomography studies in healthy subjects that reported functional mapping experiments containing a somatosensory or motor component were considered. Those investigating age, gender, disease, or drug effects were excluded. No further constraints (e.g., on acquisition and analysis details, experimental design, or stimulation procedures) were enforced. Hereby 

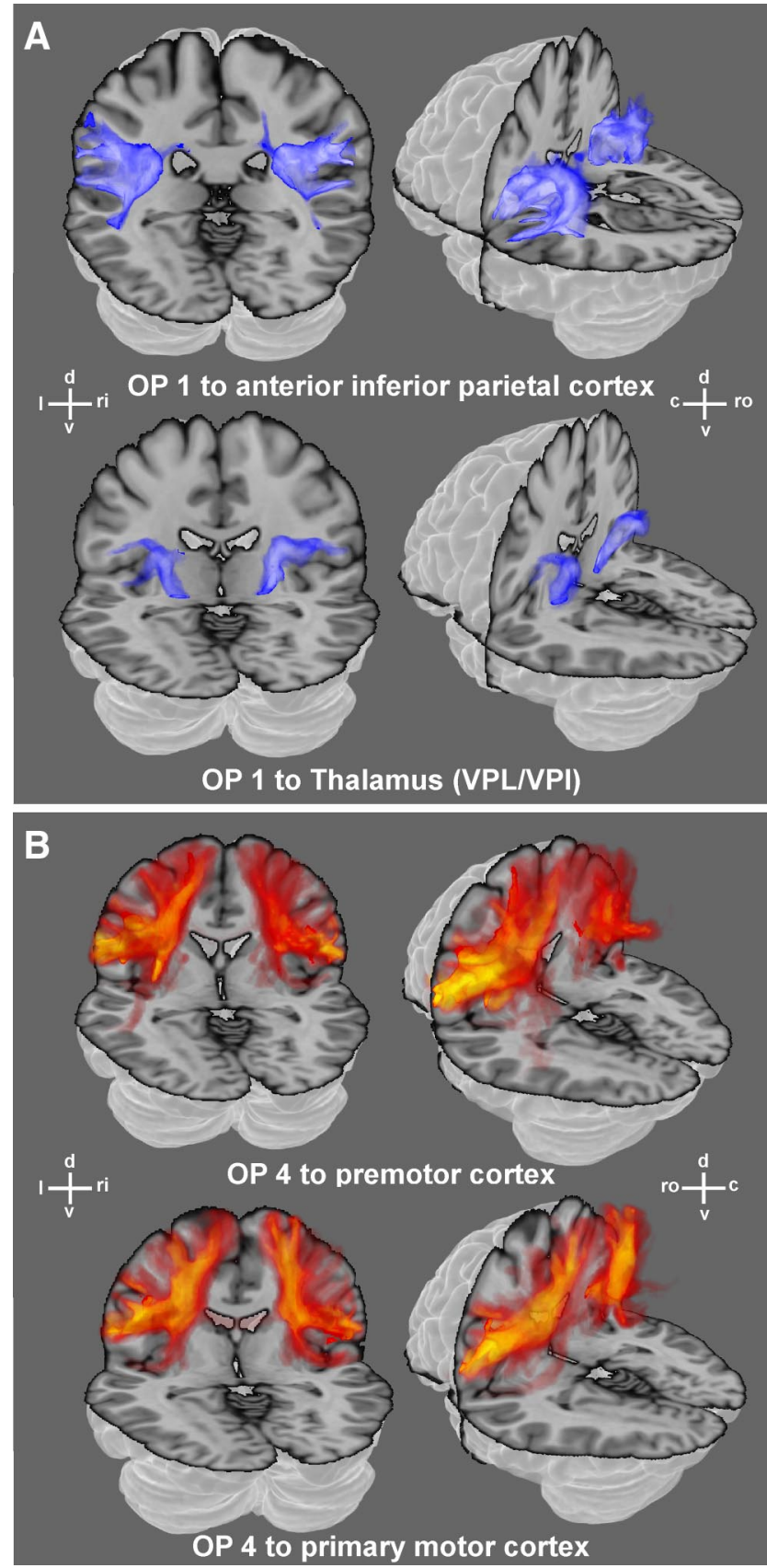

Figure 3. Examples of white matter fiber pathways as obtained from probabilistic tractography for $O P 1(\boldsymbol{A})$ and $O P 4(\boldsymbol{B})$ reconstructed in $3 \mathrm{D}$. The data shown here illustrate the tracts connecting $\mathrm{OP} 1$ and $\mathrm{OP} 4$, respectively, with exemplary target regions ( $(\mathrm{f}$. Table 1 ) and hence reveal the pathways taken by the fiber tracts connecting the seeds and targets. The absolute strength of these connections, in turn, are summarized in Figure 4. All examples are displayed on the transparent MNI single-subject template. (d: dorsal, v: ventral, l: left, ri: right, ro: rostral, c: caudal). The color scales ranging from dark to light blue and from red to yellow, respectively, denote the probability that the particular tract runs through a given voxel. Light blue or yellow indicates those locations in the white matter where the respective pathways are very likely to be found. Dark blue or red, on the other hand, indicate less likely positions of the connecting fibers.

we tried to avoid any bias in the data, but rather pool across as many different studies as possible.

Experiments that activate OP 1 or OP 4 were identified by comparing the foci reported for each of the $\sim 1500$ eligible experiments (functional mapping experiments available at the time of analysis that contained a somatosensory or motor component) in the BrainMap database to the cytoarchitectonic location of these cortical fields in the same reference space. The experiments used for the analysis of the functional connectiv-

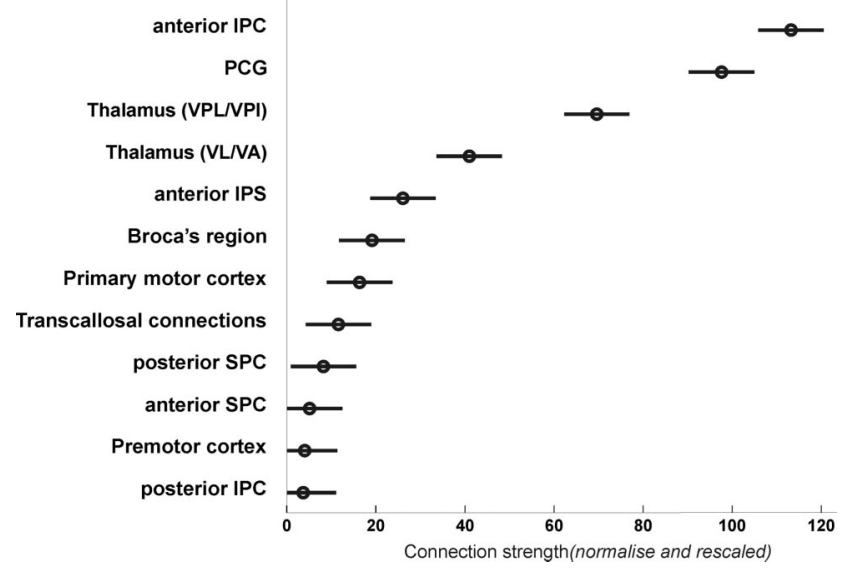

Figure 4. Mean connection strength (across subjects) between human parietal operculum (OP 1 and $O P 4$ ) and 12 different target regions assessed in the current study. Quantitative tractography was based on the sample count obtained when performing probabilistic tractography from the seed regions (OP 1 or OP 4) to the different targets (cf. Table 1 ) after these were transformed into the individual diffusion spaces of each subject. The connection probabilities obtained by this probabilistic tractography were normalized by dividing by the total connection probability of each seed and rescaled by multiplying by the mean total probability across all seeds and targets. Finally, connection densities were divided by the size of the target Vols, computed on an individual basis, and again rescaled by the mean size of all targets to provide normalized connection strengths. The circles indicate the mean connection strength of each target with the entire parietal operculum, i.e., areas $\mathrm{OP} 1$ and $\mathrm{OP} 4$. The bars denote the $95 \%$ confidence intervals on these connection strengths.

ity of OP 1 (S2) were defined by the fact that (following correction for coordinates reported according to the Talairach reference space) they featured at least one focus of activation within the volume of cortex histologically delineated as OP 1 , but no activation within the histologically delineated volume of OP 4 . Hereby, the experiments that activated OP 1 or OP 4 were objectively identified. That is, activation within our seed areas was assessed observer independently by comparing the coordinates reported for all studies within the BrainMap database to the anatomical location of cytoarchitectonically defined OP 1 and OP 4 within the same reference space, independent of how this activation was termed in the original publication. Hereby, we avoided any influence of the fact that various labels have been used for activation in the region, e.g., SII, parietal operculum, Brodmann's area (BA) 43, BA 40, parietal cortex, or subcentral gyrus. Studies activating exclusively one of these two areas (either OP 1 or OP 4) were defined by at least one reported focus in the MPM representation of this area and the absence of any reported activation focus in the respective other area or, to increase specificity, a four voxel border zone between OP 1 and OP 4 .

Given that OP 1 (S2) and OP 4 (PV) share a common border at which the face, hands, and feet are represented in either area, and acknowledging the fact that these two cortical fields are difficult to differentiate from each other functionally in nonhuman primates, the question evidently arises as to whether isolated activation in only one of these areas may be conceptually meaningful or most likely artificial. However, while S2 and $\mathrm{PV}$ tend to show concurrent activation in many experiments, there is already good evidence for differences in response properties between the various cortical fields on the parietal operculum of nonhuman primates (Robinson and Burton, 1980; Hsiao et al., 1993; Fitzgerald et al., 2004, 2006a, 2006b). Compared with electrophysiological experiments in monkeys, however, the range of tasks that may be assessed is considerably larger in human functional imaging experiments, including, in particular, experimental paradigms that investigate cognitive or affective influences on sensory-motor processing. It thus seems plausible that differences in response properties of opercular fields that have not yet been reported in monkeys may be unraveled in humans simply because the necessary paradigms are difficult to perform in animals. Moreover, differential response properties may manifest themselves as apparent shifts in somatotopic location in functional imaging data, in particular if differential 
contrasts between two conditions are considered. In this case, homogenous activation of both cortical fields by one condition may offset, leaving only an isolated peak of activation well within the cortical field that was more responsive to the other condition. This phenomenon, to which neurophysiologic mechanisms at the neuronal level may also contribute, has been discussed in great detail in a recent study by Burton et al. (2008b). It is therefore very well conceivable that isolated activations within OP 1 or OP 4 are observed in human neuroimaging data despite their close proximity and the similarities in response characteristics.

It should be noted that the seeds representing OP 1 and OP 4, respectively, in the functional connectivity analysis were defined bilaterally. This approach was based on the observation that activation of the secondary somatosensory cortex is frequently bilateral, resulting in a much reduced and ultimately insufficient sample of studies reporting unilateral activation. These, however, would be required for a separate analysis of ipsilateral and contralateral connections.

Meta-analysis algorithm. For the coordinatebased meta-analysis of neuroimaging results, we used a revised version (Eickhoff et al., 2009b) of the ALE approach (Turkeltaub et al., 2002; Laird et al., 2005). The algorithm aims at identifying areas where the convergence of activations across different experiments is higher than expected under a spatially random association between them. The key idea behind ALE is to treat the reported foci not as single points but rather as centers for 3D Gaussian probability distributions. These distributions reflect the spatial uncertainty associated with each focus, i.e., each reported set of coordinates. The width of these uncertainty functions is determined by empirical estimates of the between-subject and between-template variances encountered in neuroimaging data (Eickhoff et al., 2009b). By weighting the former by the number of subjects on which the original experiment was based, this approach moreover accommodates the notion that larger sample sizes should provide more reliable approximations of a "true" effect and should therefore be modeled by tighter Gaussian distributions yielding more localizing power (Eickhoff et al., 2009b).

The probabilities of all foci reported in a given individual experiment were combined for each voxel, resulting in a modeled activation (MA) map. Taking the union of these MA maps across all experiments yielded an ALE score for each voxel describing the convergence of results at that particular location. To distinguish true convergence between studies from random convergence, i.e., noise, these ALE scores were subsequently compared with an empirical null distribution derived from a permutation procedure (Eickhoff et al., 2009b; Caspers et al., 2010). This null distribution reflects a random spatial association between experiments but regards the within-experiment distribution of foci as fixed. Hereby, a random effects inference is invoked, focusing inference on the above chance convergence between different experiments, not the clustering of foci within a particular experiment. Computationally, deriving this null hypothesis involved sampling a voxel at random from each of the MA maps and taking the union of these values in the same manner as that done for the (spatially contingent) voxels in the actual analysis. The true ALE scores were then tested against these ALE scores obtained under the null distribution, yielding a $p$ value for each ALE score based on the proportion of equal or higher random values. The resulting nonparametric $p$ values were then transformed into $z$-scores and thresholded at a cluster level-corrected threshold of $p<0.05$ (Worsley et al., 1996).

Analysis of behavioral domain profiles. Evidently, the question also arises as to what mental processes are supported by OP 1 (S2) and OP 4 (PV) and whether the functional roles of these two parietal opercular areas differ from each other. In BrainMap, metadata are included on the cognitive, perceptual, or motor process isolated by the statistical contrast. The domain of behavioral system is classified according to five main categories: cognition, action, perception, emotion, and interoception (a complete list of the behavioral domains (BDs) in BrainMap can be accessed at http://brainmap.org/scribe/; experiments on pharmacology were excluded a priori from all analyses). We analyzed the BD metadata associated with the experiments reporting activation in OP 1 and OP 4, respectively, to determine the frequency of domain "hits" relative to its distribution across the whole brain (i.e., the entire database). In contrast to the more constrained functional connectivity analysis outlined above, this analysis was performed on the entire BrainMap database (no filter for experiments holding somatosensory or motor components). and experiments could be counted toward both regions to assemble a fully functional characterization. For each anatomical region, a $\chi^{2}$ test was performed to evaluate the regional distribution as compared with the overall database distribution (Laird et al., 2009b). If the region's distribution was significantly different, a binomial test was performed to determine which individual domains were over- or under-represented. Differences between the BD profiles of OP 1 and OP 4 were then assessed using the same approach as that for testing against a single region's profile against the database.

\section{Results}

\section{Anatomical connectivity}

Identification of white matter connections between histologically defined areas

Examples of the white matter tracts connecting the investigated areas of the parietal operculum to other regions of the frontal and parietal cortex are shown as group averages of the obtained tractography results (after normalization of the delineated tracts) in Figure 3. For each of the investigated areas, fiber tracts have been chosen that were significantly more connected to the respective area as compared with the other area (see below). 


\section{A Co-activations of the human parietal operculum} (OP1 or OP 4)



B functional connectivity

C functional vs. structural connectivity
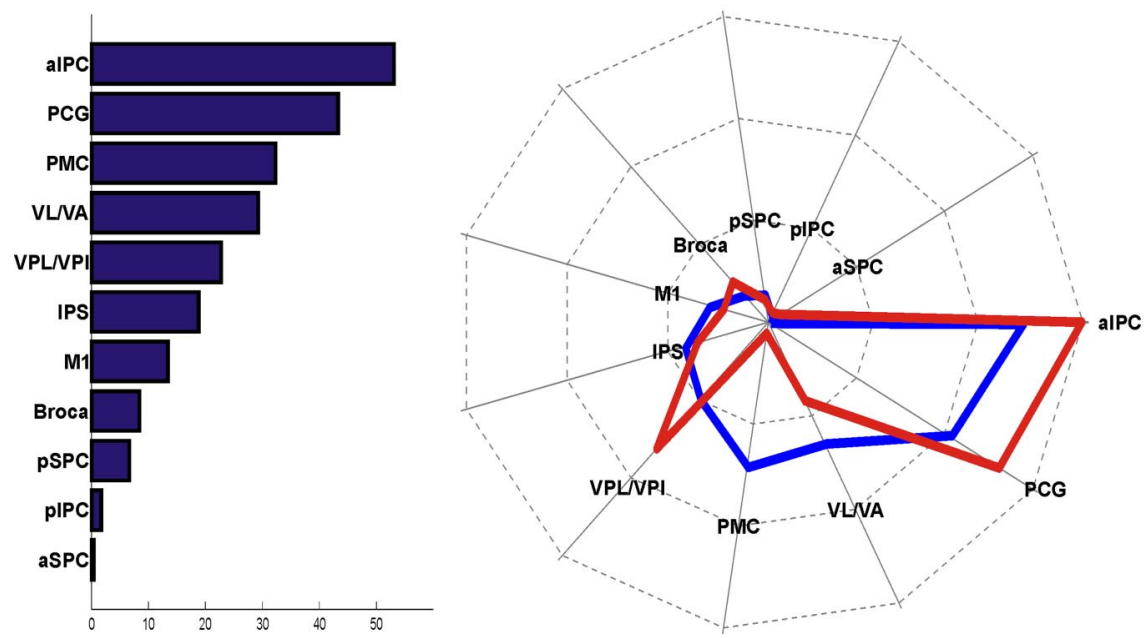

Figure 6. A, Functional connectivity of the human parietal operculum as delineated by the significant ( $p<0.05$, corrected) coactivation pattern obtained in a meta-analysis of 245 neuroimaging studies activating either OP 1 or OP 4 . The color denotes the significance of the respective results. That is, while the presence of color indicates voxels that are significantly coactivated with the seed region, the particular color indicates the strength of this effect ( $z$-score of the statistical analysis). B, Strength of functional connectivity between the human parietal operculum and the different anatomically defined targets assessed in this study (compare Fig. 4) as defined by the volume fraction of the targets' MPM representations that were significantly coactivated with OP 1 or OP 4. The labels in this graph confirm to the acronyms summarized for reference in Table 1. C, Comparison of the functional and anatomical connectivity of the human parietal operculum, i.e., OP 1 and OP 4 combined. The data used for this diagram correspond to the results shown in Figures 4 and $6 B$. However, to allow a direct comparison, the connection strengths displayed in Figures 4 and $6 B$ were rescaled to unit total connectivity, accounting for the different scaling of the data obtained from the analysis of functional and anatomical connectivity. Again, labels in this graph confirm to the acronyms explained in Table 1.

It can be noted that all fiber tracts obtained through probabilistic tractography passed through the white matter following the respective major fiber pathways and showed little variance in their course to the respective targets. OP 1 showed significantly stronger connections to anterior IPC and ventral posterior lateral nucleus/ventral posterior inferior nucleus (VPL/VPI), whereas OP 4 was significantly more strongly connected to the premotor and primary motor cortex. Most notably, although these targets neighbored each other very closely, the respective connections could be separated by means of probabilistic tractography.

Statistical analysis of connection strengths by repeated-measures ANOVA

Assessing the normalized connection probabilities by means of repeated-measures ANOVA revealed a significant main effect of the factor "target" $(F=41.77 ; p<0.001)$, indicating that the 12 assessed target regions differed from each other with respect to their anatomical connectivity with the parietal operculum (Fig. 4). Moreover, we found a significant interaction between "opercular seed" and "target" $(F=7.66 ; p<0.001)$, reflecting differences in the frontoparietal connectivity of OP 1 and OP 4 . There was, however, no significant main effect of "opercular seed," revealing that the mean connection strength of the connections to all targets does not differ significantly between OP 1 and OP 4. That is, although there is no difference between OP 1 and OP 4 with respect to the mean connection strength to all targets (absence of a significant main effect of "opercular seed"), the distribution of connection strengths to individual targets was different between OP 1 and OP 4 (significant interaction between "opercular seed" and "target"). Importantly, there was also no significant interaction with "hemisphere" $(F=0.79 ; p=0.65)$, indicating the absence of a hemispheric asymmetry in anatomical connectivity.

Anatomical connectivity of the parietal operculum (main effect across OP 1/OP 4) To resolve the significant main effect of "target," indicating that the different targets vary in their mean connection strength to OP 1 and OP 4 , a direct comparison between the different targets $(p<$ 0.05 , corrected by Tukey's method) was performed. This analysis compared the different levels of the factor "target" (i.e., the different target regions) with each other to assess which pairs differ in their mean value (i.e., connection strength of the particular area averaged across both seeds, OP 1 and OP 4). Statistical testing revealed (Fig. 4) that anterior IPC was significantly stronger connected to OP 1 and OP 4 than any other target. The differences in connectivity with OP $1 / \mathrm{OP} 4$ between the PCG and all other regions showing a lower connection density were also significant. There was no significant difference in connectivity with OP $1 / \mathrm{OP} 4$ between VPL/VPI and ventral lateral nucleus/ventral anterior nucleus (VL/VA). The connection probability from OP 1/ OP 4 toward VPL/VPI was significantly different from that toward all other areas showing a lower connectivity (Fig. 4). The same was true for VL/VA, with the exception of the connectivity to the anterior IPS. The latter area showed significantly higher connection densities than posterior IPC, premotor cortex, and both superior parietal regions. Finally, connectivity of OP 1 and OP 4 to Broca's area was significantly higher than that to the posterior IPC and the premotor cortex, respectively.

Differences in frontoparietal connectivity between $O P 1$ and $O P 4$ Follow-up comparison on the strengths of the anatomical connectivity toward the different targets between OP 1 and OP 4 revealed that the "seed $\times$ target" interaction observed in the ANOVA was based on the following significant differences (Fig. 5 ). The two regions that were most closely connected to OP 1 and OP 4, the anterior IPC and the PCG, showed a different preference with respect to their connection. While the anterior IPC features significantly higher connection probabilities with OP 1 as compared with OP 4, the PCG is significantly more densely connected to area OP 4 than to OP 1 . Besides the stronger connectivity to the anterior IPC, area OP 1 also showed significantly 
higher probabilities for anatomical connections with VPL/VPI and a significantly higher number of transcallosal connections as compared with OP 4. In contrast, area OP 4 showed significantly higher probabilities for connections with Broca's region, the primary motor cortex, and the premotor cortex than area OP 1. Finally, there was no significant difference between both seed areas with respect to their connectivity to the superior parietal cortex, the anterior IPS, the posterior IPC, and VL/VA.

In summary, OP 4 was significantly more densely connected to frontal as well as primary sensory-motor areas, while OP 1 featured higher probabilities for parietal, thalamic, and interhemispheric connections.

\section{Functional connectivity}

Searching the BrainMap database, we found 245 functional neuroimaging experiments activating either OP 1 or OP 4. Of these, 80 experiments exclusively activated OP 1 (but featured no focus in OP 4 or the border zone), and another 61 reported activation only in OP 4.

\section{Functional connectivity of the parietal} operculum (main effect across OP 1/OP 4) The functional connectivity of the human parietal operculum was revealed by significant coactivations in all those studies that activated either of the two areas as shown in Figure 6A. As expected, coactivations and hence functional connectivity to OP 1 and OP 4 were found in a widely distributed frontoparietal network. Comparison of significant coactivation with the same target VOIs as those used for the DTI analysis (Table 1) yielded the following results (Fig. 6B). Functional connectivity was most prevalent in anterior IPC (53.1\% of the voxels in this target VOI showing significant functional connectivity), followed by the PCG (43.3\% of this target VOI showing significant functional connectivity), the premotor cortex (32.3\%), and VL/VA (29.4\%). The VPL/VPI (22.5\%), the anterior IPS (18.8\%), and the primary motor cortex $(13.4 \%)$ showed intermediate functional connectivity, while Broca's region $(8.4 \%)$ and in particular posterior superior parietal cortex (SPC) (6.6\%), posterior IPC (1.7\%), and anterior SPC $(0.4 \%)$ showed little coactivation with our seed regions (OP 1/OP 4). Comparing this distribution of functional connectivity with the respective anatomical connectivity strengths, both similarities and differences become evident. (Fig. 6C). In particular, it can be noted that the regions featuring the strongest anatomical connections (anterior IPC, PCG) with the seed region on the parietal operculum defined by cytoarchitectonic areas OP 1 and OP 4 also show the highest functional connectivity. In contrast, both anatomical and functional connectivity with regions like anterior and posterior SPC and posterior IPC are congruently low. Notable exceptions, however, can be found with respect to the connectivity of OP1 and OP 4 with the premotor cortex and
VL/VA. Here, functional connectivity is much stronger as compared with the density of anatomical connections.

Differences in frontoparietal functional connectivity between $O P 1$ and $O P 4$

To assess the functional connectivity of OP 1 and OP 4, separate meta-analyses were performed including only those studies that specifically activated the respective area. The resulting coactivation maps (Fig. $7 A, B$ ) show patterns of functional connectivity that, albeit sharing some similarities, are distinctly different from each other. In particular, it can be noted, that OP 1 activations are primarily associated with activity of the anterior IPC, extending into the IPS. Regions coactivating with OP 4, on the other hand, were found anterior to those and consisted of the inferior frontal and pericentral somatosensory and motor cortices. Concurrent coactivation, finally, was in particular found bilaterally in the thalamus (with a focus on VL/VA known to project also strongly to the premotor and primary motor cortices), in the region of the supplementary motor cortex on the mesial wall of the frontal lobe and inferior frontal gyri on both hemispheres. 

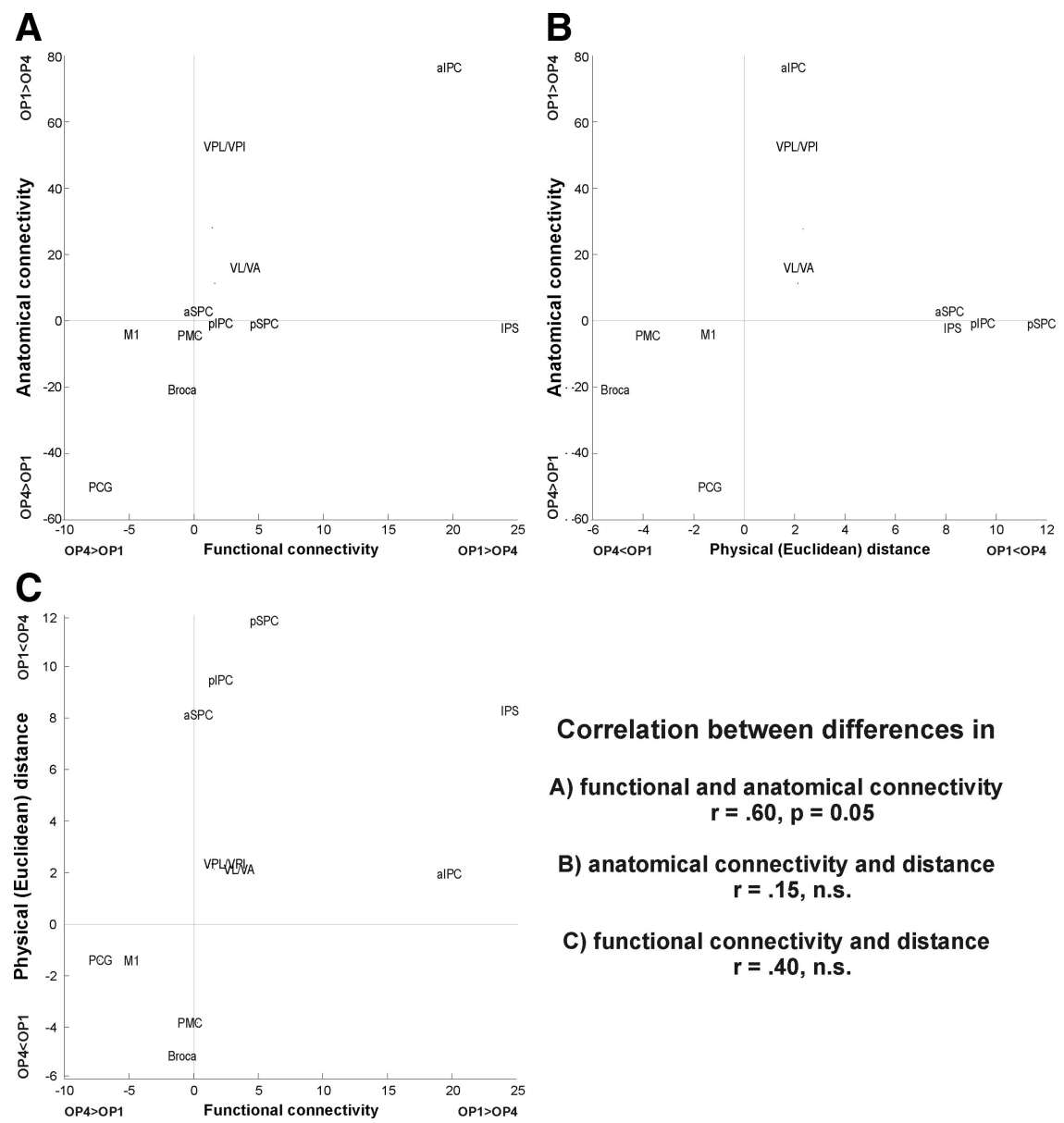

Correlation between differences in

A) functional and anatomical connectivity $r=.60, p=0.05$

B) anatomical connectivity and distance $r=.15$, n.s.

C) functional connectivity and distance $r=.40$, n.s.

Figure 8. A, Comparison of the differences between $O P 1$ and $O P 4$ in terms of their anatomical (as assessed by probabilistic tractography) and functional (as assessed by meta-analysis of neuroimaging data) connectivity to the assessed frontoparietal targets. Each target is represented by a data point indicated by the position of its acronym on a two-dimensional coordinate grid. The $x$-coordinate of this point indicates the difference in functional connectivity (quantified by coactivated volume fraction) between this target and OP 1 on one hand and OP 4 on the other. Precisely, the $x$-value of target acronym's position is equivalent to the strength of functional connectivity between this target and $\mathrm{OP} 1$ minus the strength of functional connectivity between this target and $\mathrm{OP} 4$. The $y$-coordinate indicates the difference in anatomical connectivity (quantified by normalized connection strength). That is, the $y$-value of target acronym's position is equivalent to the strength of anatomical connectivity from OP 1 and this target minus the strength of anatomical connectivity from OP 4 and this target. It may be argued that one or both of these measures may be confounded by the physical distance between seed and target. Hence, differences in anatomical and functional connectivity were also plotted against the difference between OP 1 and OP 4 in their physical distance (quantified by the mean Euclidean distance across seed voxels to the nearest target voxel) to the respective target. $\boldsymbol{B}$ and $\boldsymbol{C}$, The comparison between anatomical connectivity and physical (Euclidean) distance is shown in $\boldsymbol{B}$, and the comparison between functional connectivity and physical distance is shown in $\boldsymbol{C}$. It can be seen that differences between OP 1 and OP 4 in terms of functional and anatomical connectivity are significantly correlated to each other but not to physical distances to the targets.

To identify differences in functional connectivity between OP 1 and OP 4, we then contrasted the two functional connectivity maps (Laird et al., 2005), hereby delineating those voxels that showed significantly ( $p<0.05$ corrected) higher probability of coactivation with OP 1 and OP 4, respectively (Fig. 7C). Significant differences were found in the parietal operculum, which is a trivial finding as the two cohorts of experiments being compared were defined by the presence of activations in OP 1 and OP 4, respectively. However, we also found several other regions showing significant differences in functional connectivity with OP 1 and OP 4, respectively. In particular, activation in OP 1 was significantly stronger associated with coactivations in anterior IPC, where $45.5 \%$ of all voxels showing significantly higher functional connectivity with OP 1 were located. Moreover, $35.2 \%$ of the significantly different voxels were localized in anterior IPS, and $18.5 \%$ were allocated to the PCG. Regions show- ing significantly higher functional connectivity with $\mathrm{OP} 4$, in turn, were the PCG (where $68.8 \%$ of the voxels showing significantly higher connectivity to OP 4 were located), primary motor cortex (18.9\%), and premotor cortex (10.3\%).

\section{Comparison between anatomical and functional connectivity}

These differences in functional connectivity of OP 1 and OP 4 to other frontoparietal areas relate well to the above-mentioned differences in anatomical connectivity between these two areas (Fig. 8). It can be noted that those target regions that have a stronger anatomical connectivity to OP 1 as compared with OP 4, e.g., the anterior IPC or the thalamus, also tend to have a higher functional connectivity with that region. In contrast, regions like M1 or Broca's area, which are more closely connected to OP 4 than to OP 1 , also feature more frequent coactivations with OP 4. Finally, those regions that show little difference in anatomical connectivity coactivate at about the same amount with both areas. Consequently, there is a significant ( $p=0.05, r=0.60)$ correlation between both measures of connectivity.

Besides these congruencies, however, there also exist few notable exceptions. For example, whereas there is little difference in anatomical connectivity to IPS between OP 1 and OP 4, OP 1 features a considerably stronger functional connectivity to this region. Likewise, whereas VPL/VPI are anatomically much more strongly connected to OP 1 as compared with OP 4, the corresponding difference in functional connectivity is considerably smaller.

To exclude a potential influence of the distance between different areas, we assessed how these differences in anatomical and functional connectivity relate to differences in the proximity, i.e., physical distance, between OP 1 and OP 4, respectively, and the different targets. As shown in Figure 8, differences in physical distance are not correlated to differences in anatomical or functional connectivity.

\section{Analysis of behavioral domain profiles}

Assessing the BD meta-data associated with all experiments in the BrainMap database that featured at least one focus of activation in OP 1 and OP 4, respectively, indicated that the BD profiles of both cortical fields were significantly different from the overall distribution in the BrainMap database (Fig. 9). In particular, "action" was significantly over-represented in both parietal opercular regions. That is, a higher proportion of experiments that feature activation in OP 1/OP 4 relate to action as expected from the overall proportion of "action" experiments in the BrainMap database. "Emotion" and "cognition," in contrast, were underrepresented in both assessed cortical fields, whereas there was no 


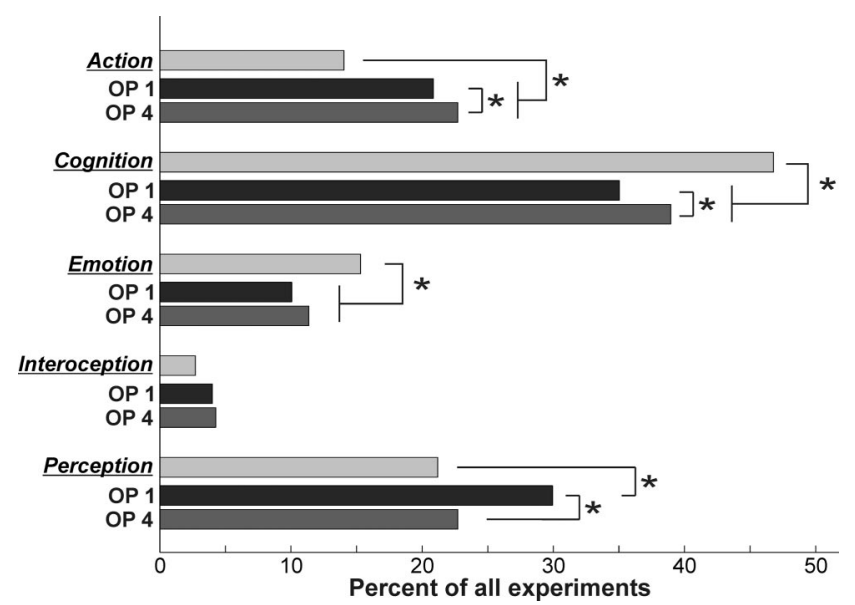

Figure 9. Behavioral domain profiles for all those experiments that feature at least one activation in OP 1 and OP 4, respectively. BrainMap counts (light gray histograms) represent the proportion of experiments in BrainMap that relate to the particular BD category. Dark gray and medium gray histograms represent the proportion of experiments featuring an activation in $\mathrm{OP} 1$ and $\mathrm{OP} 4$, respectively, that belong to the particular $\mathrm{BD}$. All histograms were significantly different from each other with respect to overall shape. Asterisks denote significant $(p<0.05)$ differences in the individual comparisons.

significant difference between OP 1 or OP 4 and the entire database with respect to experiments relating to interoception. Finally, only in OP 1, but not in OP 4, the "perception" BD was significantly over-represented.

There was also a significant difference in the BD profiles of experiments featuring activation in OP 1 and OP 4, respectively. This effect related to a higher proportion of "action" and "cognition" experiments found among those that activate OP 4. Among those experiments that activate OP 1, however, the proportion of those related to "perception" and in particular "somesthesis" was significantly higher than in OP 4.

\section{Discussion}

In the present study, anatomical and functional connectivity of human parietal opercular areas OP 1 and OP 4 were investigated by probabilistic tractography and coordinate-based metaanalysis. Anatomical and functional connectivity of these two areas, considered together, was closest with the anterior IPC, PCG, and the thalamus. Particularly the PMC, however, showed stronger functional than anatomical connectivity to the parietal operculum. Probabilistic tractography and coactivation mapping revealed largely congruent differences (across methods) between OP 1 and OP 4 connectivity. OP 1 is closer connected to anterior IPC, the IPS, VPL/VPI, and the opposite hemisphere. OP 4, in turn, has closer anatomical and functional connections to the PCG and M1 as well as premotor and inferior frontal cortices.

\section{Comparison to connectivity in nonhuman primates}

Macaque areas S2 and PV have dense reciprocal connections to the PCG (particularly areas $3 \mathrm{~b}$ and 1 ) and inferior parietal area $7 \mathrm{~b}$ (Disbrow et al., 2003), while marmosets feature a similar yet less specific pattern of connectivity (Krubitzer and Kaas, 1990; Qi et al., 2002). We here confirm the close connectivity of S2 (OP 1) and PV (OP 4) to the PCG and the inferior parietal cortex in humans. The dissociation reported here between S2 (connected to inferior parietal cortex) and PV (connected to the PCG) has not been found in any other primate species.

Area OP 4 (PV) featured significantly higher connectivity to the PMC and Broca's region than OP 1. Our data hence match observations of axonal connectivity between PMC and PV but not S2 in macaques (Disbrow et al., 2003). In marmosets, PMC connectivity of area S2 was reported but weaker than that of area PV (Stepniewska et al., 2006) While Disbrow et al. (2003) found no evidence for M1 connectivity of S2 or PV in macaques, an earlier study reported strong connectivity of a seed region comprising both areas with PMC and M1 (Cipolloni and Pandya, 1999). In marmosets, connections between M1 and PV (but not S2) have also been demonstrated (Qi et al., 2002). The human data fit this somewhat inconsistent picture by showing significantly stronger connectivity of OP 4 (PV) to all frontal targets, although the dissociation between anatomical and functional connectivity to the PMC and open questions about homologies warrant further investigation.

In macaques (Friedman and Murray, 1986; Disbrow et al., 2002, 2003) and marmosets (Krubitzer and Kaas, 1990; Qi et al., 2002), S2 and PV receive their main thalamic input from the ventroposterior inferior nucleus (VPI), while PV was reported to receive input from nuclei associated with the motor system (e.g., VL). Within the limits of resolution achievable by in vivo imaging methods, the current analysis confirms these findings in humans.

\section{Criteria for cortical areas}

A sensory cortical area should be defined by the following criteria (Kaas, 1983; Orban et al., 2004): (1) a distinct histology, as shown for S2/PV in marmosets (Krubitzer and Kaas, 1990) and macaques (Krubitzer et al., 1995), as well as for OP 1 (S2)/OP 4 (PV) in humans (Eickhoff et al., 2006c); (2) a distinct pattern of connectivity, as demonstrated for S2/PV in marmosets (Krubitzer and Kaas, 1990; Qi et al., 2002) and macaques (Burton et al., 1995; Disbrow et al., 2002, 2003) and reported here for OP 1/OP 4 in humans; (3) the presence of a somatotopic map, as demonstrated for S2 (OP 1)/PV (OP 4) in marmosets (Krubitzer and Kaas, 1990; Qi et al., 2002), macaques (Krubitzer et al., 1995; Disbrow et al., 2003), and humans (Eickhoff et al., 2007); (4) distinct functional properties. Up to now, data on the functional differentiation between S2 (OP 1) and PV (OP 4) are limited but growing in nonhuman primates (Fitzgerald et al., 2004, 2006b) and man (Burton et al., 2008a, 2008b) as discussed below.

\section{Potential functional roles for OP 1/S2 and OP 4/PV}

OP 4 is densely connected to the PCG and the frontal cortex and may consequently play a role in sensory-motor integration processes, such as incorporating sensory feedback into motor actions (Rizzolatti and Wolpert, 2005; Halsband and Lange, 2006). Knowledge of performed movements is also crucial in tactile object recognition and manipulation. Both roles have previously been ascribed to the parietal operculum (Inoue et al., 2002; Wasaka et al., 2005). Based on the current results, we suggest that they may be sustained particularly by OP 4 . This interpretation is supported by studies showing that activity in OP 4 but not OP 1 is modulated by increased attention during tactile object discrimination (Young et al., 2004), and that activation to active discrimination tasks activated a more anterior focus than a passive somatosensory control task (Ledberg et al., 1995). In a series of studies, Fitzgerald and colleagues described several functionally distinct fields on the parietal operculum of macaque monkeys (Fitzgerald et al., 2004, 2006a, 2006b). They noted that neurons in fields proposed to match PV may relate to motor functions, as these showed responses similar to the hand manipulation neurons in the superior parietal cortex (Kalaska et al., 1983). Moreover, processing of proprioceptive input and integration of information across multiple digits favored the hypothesis that PV 
may extract structural components of actively touched objects, which could then spatially guide further manipulation (Burton et al., 1997, 1999).

In contrast, strong connectivity of OP 1 with anterior parietal cortex, VPL/VPI, and the contralateral hemisphere may predispose it to perform more integrative aspects of somatosensory processing. Hence, OP 1 may represent the anatomical substrate of the various more complex functions reported to involve the parietal operculum, such as tactile working memory, stimulus discrimination (particularly frequency), and perceptual learning (Romo et al., 2002; Torquati et al., 2002; Pleger et al., 2003; Burton et al., 2008a, 2008b). Matching this view, neurons showing attention and stimulus discrimination have been described in the posterior parietal operculum (area S2) of macaque monkeys (Robinson and Burton, 1980; Hsiao et al., 1993). It is not yet precisely known how these functions (attention and stimulus discrimination) are implemented neuronally. Evidence from single-cell recordings, however, suggests that differences between parietal opercular fields with respect to modulations of synchrony, receptive field layout, and neuronal tuning may underlie their functional differentiation (Steinmetz et al., 2000; Fitzgerald et al., 2006a). Finally, OP 1 may also hold an important role in bimanual processing. In an fMRI/MEG study, Disbrow et al. (2001) demonstrated that S2/PV receives bilateral input, but extensive contralateral processing occurs before interhemispheric transfer. We propose that particularly the later ipsilateral component might be attributed to OP 1, given the higher amount of transcallosal connections in this area. This would match the observation that unilateral stimulation activation on the anterior parietal operculum (presumably OP 4) is followed by ipsilateral posterior activation (presumably OP 1) with a latency of $\sim 30 \mathrm{~ms}$ (Mima et al., 1997).

Assessing the behavioral domain profiles of experiments featuring activation in OP 1 (S2) and OP 4 (PV), respectively, were well in accordance with the differentiation suggested by the differences in anatomical and functional connectivity, as well as the hypothesis from nonhuman primate data. This analysis of functional processes isolated by the statistical contrasts that activated the two parietal opercular areas confirmed that OP 1 (S2) was indeed more likely activated by somatosensory perceptive tasks, whereas OP 4 was more associated with action, i.e., motorrelated experiments.

\section{Discrepancies between connectivity measures}

Despite the close congruency between anatomical and functional connectivity (Figs. 6C, 8), several divergences were noted. These discrepancies may have resulted from various sources of measurement error and noise in the data that may differ systematically or unsystematically between both approaches. Examples for such methodological issues would be discrepancies in the effective spatial resolution of the data, differences in assessed sample, influences of the choices about the used models for diffusion tractography or functional connectivity analyses (as various methods exist for either approach), differences in potential confounds affecting the two very different approaches, or potentially dissimilar characteristics of unsystematic noise.

However, it should be noted that there are also major conceptual differences between functional and anatomical connectivity, as these assess different properties of brain networks. That is, there are several theoretical reasons, discussed below, why these two approaches may not provide completely congruent results even if there is no systematic or unsystematic error due to methodological or technical issues.
Coactivation of two regions may not be mediated by direct anatomical connections but via additional structures, e.g., relaying information from VL/VA or the premotor cortex to OP 4. Relay processes, however, could also be transmitted through cascades of several intermediates or via cortical-subcortical loops (Grefkes et al., 2008b; Eickhoff et al., 2009a).

A third area could induce correlated activation in regions not anatomically connected. That is, functional connectivity may be driven by an external source inducing concurrent activity in both areas, e.g., stimulus-driven activity in early sensory areas that is forwarded to parietal opercular areas for perceptual analysis and, in parallel, to premotor cortex for response preparation.

A very weak anatomical connection between two regions may still hold a high functional significance (Friston, 2002; Grefkes et al., 2008a), e.g., if one area's activity depends on a "go-signal" from another region. Functional connectivity is hence strongly influenced not only by the strength of an anatomical connection but also by the information conveyed through it.

None of these mechanisms inducing functional coupling would be reflected in anatomical connectivity measured by DTI. A deeper understanding of brain connectivity and the ensuing networks should thus rely on a combination of different but complementary approaches.

\section{References}

Amunts K, Schleicher A, Burgel U, Mohlberg H, Uylings HB, Zilles K (1999) Broca's region revisited: cytoarchitecture and intersubject variability. J Comp Neurol 412:319-341.

Amunts K, Weiss PH, Mohlberg H, Pieperhoff P, Eickhoff S, Gurd JM, Marshall JC, Shah NJ, Fink GR, Zilles K (2004) Analysis of neural mechanisms underlying verbal fluency in cytoarchitectonically defined stereotaxic space-the roles of Brodmann areas 44 and 45 . Neuroimage 22:42-56.

Ashburner J, Friston KJ (2005) Unified segmentation. Neuroimage 26:839-851.

Behrens TE, Johansen-Berg H, Woolrich MW, Smith SM, Wheeler-Kingshott CA, Boulby PA, Barker GJ, Sillery EL, Sheehan K, Ciccarelli O, Thompson AJ, Brady JM, Matthews PM (2003a) Non-invasive mapping of connections between human thalamus and cortex using diffusion imaging. Nat Neurosci 6:750-757.

Behrens TE, Woolrich MW, Jenkinson M, Johansen-Berg H, Nunes RG, Clare S, Matthews PM, Brady JM, Smith SM (2003b) Characterization and propagation of uncertainty in diffusion-weighted MR imaging. Magn Reson Med 50:1077-1088.

Behrens TE, Berg HJ, Jbabdi S, Rushworth MF, Woolrich MW (2007) Probabilistic diffusion tractography with multiple fibre orientations: what can we gain? Neuroimage 34:144-155.

Burton H, Fabri M, Alloway K (1995) Cortical areas within the lateral sulcus connected to cutaneous representations in areas $3 \mathrm{~b}$ and 1 : a revised interpretation of the second somatosensory area in macaque monkeys. J Comp Neurol 355:539-562.

Burton H, MacLeod AM, Videen TO, Raichle ME (1997) Multiple foci in parietal and frontal cortex activated by rubbing embossed grating patterns across fingerpads: a positron emission tomography study in humans. Cereb Cortex 7:3-17.

Burton H, Abend NS, MacLeod AM, Sinclair RJ, Snyder AZ, Raichle ME (1999) Tactile attention tasks enhance activation in somatosensory regions of parietal cortex: a positron emission tomography study. Cereb Cortex 9:662-674.

Burton H, Sinclair RJ, McLaren DG (2008a) Cortical network for vibrotactile attention: a fMRI study. Hum Brain Mapp 29:207-221.

Burton H, Sinclair RJ, Wingert JR, Dierker DL (2008b) Multiple parietal operculum subdivisions in humans: tactile activation maps. Somatosens Mot Res 25:149-162.

Caspers S, Geyer S, Schleicher A, Mohlberg H, Amunts K, Zilles K (2006) The human inferior parietal cortex: cytoarchitectonic parcellation and interindividual variability. 33:430-448.

Caspers S, Eickhoff SB, Geyer S, Scheperjans F, Mohlberg H, Zilles K, Amunts 
K (2008) The human inferior parietal lobule in stereotaxic space. Brain Struct Funct 212:481-495.

Caspers S, Zilles K, Laird AR, Eickhoff SB (2010) ALE meta-analysis of action observation and imitation in the human brain. Neuroimage 50: $1148-1167$.

Choi HJ, Zilles K, Mohlberg H, Schleicher A, Fink GR, Armstrong E, Amunts K (2006) Cytoarchitectonic identification and probabilistic mapping of two distinct areas within the anterior ventral bank of the human intraparietal sulcus. J Comp Neurol 495:53-69.

Cipolloni PB, Pandya DN (1999) Cortical connections of the frontoparietal opercular areas in the rhesus monkey. J Comp Neurol 403:431-458.

Collins DL, Neelin P, Peters TM, Evans AC (1994) Automatic 3D intersubject registration of MR volumetric data in standardized Talairach space. J Comput Assist Tomogr 18:192-205.

Coq JO, Qi H, Collins CE, Kaas JH (2004) Anatomical and functional organization of somatosensory areas of the lateral fissure of the New World titi monkey (Callicebus moloch). J Comp Neurol 476:363-387.

Cusick CG, Wall JT, Felleman DJ, Kaas JH (1989) Somatotopic organization of the lateral sulcus of owl monkeys: area $3 \mathrm{~b}, \mathrm{~S}-\mathrm{II}$, and a ventral somatosensory area. J Comp Neurol 282:169-190.

Disbrow E, Roberts T, Krubitzer L (2000) Somatotopic organization of cortical fields in the lateral sulcus of Homo sapiens: evidence for SII and PV. J Comp Neurol 418:1-21.

Disbrow E, Roberts T, Poeppel D, Krubitzer L (2001) Evidence for interhemispheric processing of inputs from the hands in human S2 and PV. J Neurophysiol 85:2236-2244.

Disbrow E, Litinas E, Recanzone GH, Slutsky D, Krubitzer L (2002) Thalamocortical connections of the parietal ventral area (PV) and the second somatosensory area (S2) in macaque monkeys. Thalamus Relat Syst 1:289-302.

Disbrow E, Litinas E, Recanzone GH, Padberg J, Krubitzer L (2003) Cortical connections of the second somatosensory area and the parietal ventral area in macaque monkeys. J Comp Neurol 462:382-399.

Eickhoff SB, Stephan KE, Mohlberg H, Grefkes C, Fink GR, Amunts K, Zilles K (2005) A new SPM toolbox for combining probabilistic cytoarchitectonic maps and functional imaging data. Neuroimage 25:1325-1335.

Eickhoff SB, Amunts K, Mohlberg H, Zilles K (2006a) The human parietal operculum. II. Stereotaxic maps and correlation with functional imaging results. Cereb Cortex 16:268-279.

Eickhoff SB, Heim S, Zilles K, Amunts K (2006b) Testing anatomically specified hypotheses in functional imaging using cytoarchitectonic maps. Neuroimage 32:570-582.

Eickhoff SB, Lotze M, Wietke B, Amunts K, Enck P, Zilles K (2006c) Segregation of visceral and somatosensory afferents: an fMRI and cytoarchitectonic mapping study. Neuroimage 31:1004-1014.

Eickhoff SB, Schleicher A, Zilles K, Amunts K (2006d) The human parietal operculum. I. Cytoarchitectonic mapping of subdivisions. Cereb Cortex 16:254-267.

Eickhoff SB, Weiss PH, Amunts K, Fink GR, Zilles K (2006e) Identifying human parietal-insular vestibular cortex using fMRI and cytoarchitectonic mapping. Hum Brain Mapp 27:611-621.

Eickhoff SB, Grefkes C, Zilles K, Fink GR (2007) The somatotopic organization of cytoarchitectonic areas on the human parietal operculum. Cereb Cortex 17:1800-1811.

Eickhoff SB, Heim S, Zilles K, Amunts K (2009a) A systems perspective on the effective connectivity of overt speech production. Philos Transact A Math Phys Eng Sci 367:2399-2421.

Eickhoff SB, Laird AR, Grefkes C, Wang LE, Zilles K, Fox PT (2009b) Coordinate-based activation likelihood estimation meta-analysis of neuroimaging data: a random-effects approach based on empirical estimates of spatial uncertainty. Hum Brain Mapp 30:2907-2926.

Evans AC, Marrett S, Neelin P, Collins L, Worsley K, Dai W, Milot S, Meyer E, Bub D (1992) Anatomical mapping of functional activation in stereotactic coordinate space. Neuroimage 1:43-53.

Fitzgerald PJ, Lane JW, Thakur PH, Hsiao SS (2004) Receptive field properties of the macaque second somatosensory cortex: evidence for multiple functional representations. J Neurosci 24:11193-11204.

Fitzgerald PJ, Lane JW, Thakur PH, Hsiao SS (2006a) Receptive field properties of the macaque second somatosensory cortex: representation of orientation on different finger pads. J Neurosci 26:6473-6484.

Fitzgerald PJ, Lane JW, Thakur PH, Hsiao SS (2006b) Receptive field (RF) properties of the macaque second somatosensory cortex: RF size, shape, and somatotopic organization. J Neurosci 26:6485-6495.

Fox PT, Lancaster JL (2002) Opinion: Mapping context and content: the BrainMap model. Nat Rev Neurosci 3:319-321.

Friedman DP, Murray EA (1986) Thalamic connectivity of the second somatosensory area and neighboring somatosensory fields of the lateral sulcus of the macaque. J Comp Neurol 252:348-373.

Friston K (2002) Functional integration and inference in the brain. Prog Neurobiol 68:113-143.

Geyer S (2003) The microstructural border between the motor and the cognitive domain in the human cerebral cortex. Wien: Springer.

Geyer S, Ledberg A, Schleicher A, Kinomura S, Schormann T, Burgel U, Klingberg T, Larsson J, Zilles K, Roland PE (1996) Two different areas within the primary motor cortex of man. Nature 382:805-807.

Geyer S, Schleicher A, Zilles K (1999) Areas 3a, 3b, and 1 of human primary somatosensory cortex. 1. Microstructural organization and interindividual variability. Neuroimage 10:63-83.

Geyer S, Schormann T, Mohlberg H, Zilles K (2000) Areas 3a, 3b, and 1 of human primary somatosensory cortex. Part 2 . Spatial normalization to standard anatomical space. Neuroimage 11:684-696.

Grefkes C, Geyer S, Schormann T, Roland P, Zilles K (2001) Human somatosensory area 2: observer-independent cytoarchitectonic mapping, interindividual variability, and population map. Neuroimage 14:617631.

Grefkes C, Eickhoff SB, Nowak DA, Dafotakis M, Fink GR (2008a) Dynamic intra- and interhemispheric interactions during unilateral and bilateral hand movements assessed with fMRI and DCM. Neuroimage 41:1382-1394.

Grefkes C, Nowak DA, Eickhoff SB, Dafotakis M, Kust J, Karbe H, Fink GR (2008b) Cortical connectivity after subcortical stroke assessed with functional magnetic resonance imaging. Ann Neurol 63:236-246.

Halsband U, Lange RK (2006) Motor learning in man: a review of functional and clinical studies. J Physiol Paris 99:414-424.

Hsiao SS, O’Shaughnessy DM, Johnson KO (1993) Effects of selective attention on spatial form processing in monkey primary and secondary somatosensory cortex. J Neurophysiol 70:444-447.

Huffman KJ, Nelson J, Clarey J, Krubitzer L (1999) Organization of somatosensory cortex in three species of marsupials, Dasyurus hallucatus, Dactylopsila trivirgata, and Monodelphis domestica: neural correlates of morphological specializations. J Comp Neurol 403:5-32.

Inoue K, Yamashita T, Harada T, Nakamura S (2002) Role of human SII cortices in sensorimotor integration. Clin Neurophysiol 113:1573-1578.

Jung P, Baumgärtner U, Stoeter P, Treede RD (2009) Structural and functional asymmetry in the human parietal opercular cortex. J Neurophysiol 101:3246-3257.

Kaas JH (1983) What, if anything, is SI? Organization of first somatosensory area of cortex. Physiol Rev 63:206-231.

Kaas JH, Collins CE (2003) The organization of somatosensory cortex in anthropoid primates. Adv Neurol 93:57-67.

Kalaska JF, Caminiti R, Georgopoulos AP (1983) Cortical mechanisms related to the direction of two-dimensional arm movements: relations in parietal area 5 and comparison with motor cortex. Exp Brain Res 51:247-260.

Kell CA, von Kriegstein K, Rösler A, Kleinschmidt A, Laufs H (2005) The sensory cortical representation of the human penis: revisiting somatotopy in the male homunculus. J Neurosci 25:5984-5987.

Koski L, Paus T (2000) Functional connectivity of the anterior cingulate cortex within the human frontal lobe: a brain-mapping meta-analysis. Exp Brain Res 133:55-65.

Krubitzer LA, Calford MB (1992) Five topographically organized fields in the somatosensory cortex of the flying fox: microelectrode maps, myeloarchitecture, and cortical modules. J Comp Neurol 317:1-30.

Krubitzer LA, Kaas JH (1990) The organization and connections of somatosensory cortex in marmosets. J Neurosci 10:952-974.

Krubitzer L, Clarey J, Tweedale R, Elston G, Calford M (1995) A redefinition of somatosensory areas in the lateral sulcus of macaque monkeys. J Neurosci 15:3821-3839.

Laird AR, Fox PM, Price CJ, Glahn DC, Uecker AM, Lancaster JL, Turkeltaub PE, Kochunov P, Fox PT (2005) ALE meta-analysis: controlling the false discovery rate and performing statistical contrasts. Hum Brain Mapp 25:155-164.

Laird AR, Eickhoff SB, Kurth F, Fox PM, Uecker AM, Turner JA, Robinson JL, 
Lancaster JL, Fox PT (2009a) ALE meta-analysis workflows via the brainmap database: progress towards a probabilistic functional brain atlas. Front Neuroinformatics 3:23.

Laird AR, Eickhoff SB, Li K, Robin DA, Glahn DC, Fox PT (2009b) Investigating the functional heterogeneity of the default mode network using coordinate-based meta-analytic modeling. J Neurosci 29:14496-14505.

Ledberg A, O’Sullivan BT, Kinomura S, Roland PE (1995) Somatosensory activations of the parietal operculum of man: a PET study. Eur J Neurosci 7:1934-1941.

Mima T, Ikeda A, Nagamine T, Yazawa S, Kunieda T, Mikuni N, Taki W, Kimura J, Shibasaki H (1997) Human second somatosensory area: subdural and magnetoencephalographic recording of somatosensory evoked responses. J Neurol Neurosurg Psychiatry 63:501-505.

Orban GA, Van ED, Vanduffel W (2004) Comparative mapping of higher visual areas in monkeys and humans. Trends Cogn Sci 8:315-324.

Pleger B, Foerster AF, Ragert P, Dinse HR, Schwenkreis P, Malin JP, Nicolas V, Tegenthoff M (2003) Functional imaging of perceptual learning in human primary and secondary somatosensory cortex. Neuron 40:643653.

Qi HX, Lyon DC, Kaas JH (2002) Cortical and thalamic connections of the parietal ventral somatosensory area in marmoset monkeys (Callithrix jacchus). J Comp Neurol 443:168-182.

Rizzolatti G, Wolpert DM (2005) Motor systems. Curr Opin Neurobiol 15:623-625

Robinson CJ, Burton H (1980) Somatic submodality distribution within the second somatosensory (SII), 7b, retroinsular, postauditory, and granular insular cortical areas of M. fascicularis. J Comp Neurol 192:93-108.

Robinson JL, Laird AR, Glahn DC, Lovallo WR, Fox PT (2009) Metaanalytic connectivity modeling: delineating the functional connectivity of the human amygdala. Hum Brain Mapp 31:173-184.

Romo R, Hernandez A, Zainos A, Lemus L, Brody CD (2002) Neuronal correlates of decision-making in secondary somatosensory cortex. Nat Neurosci 5:1217-1225.

Rushworth MF, Behrens TE, Johansen-Berg H (2006) Connection patterns distinguish 3 regions of human parietal cortex. Cereb Cortex 16:14181430.

Scheperjans F, Hermann K, Eickhoff SB, Amunts K, Schleicher A, Zilles K (2008a) Observer-independent cytoarchitectonic mapping of the human superior parietal cortex. Cereb Cortex 18:846-867.

Scheperjans F, Eickhoff SB, Homke L, Mohlberg H, Hermann K, Amunts K, Zilles K (2008b) Probabilistic maps, morphometry, and variability of cytoarchitectonic areas in the human superior parietal cortex. Cereb Cortex 18:2141-2157.

Schleicher A, Palomero-Gallagher N, Morosan P, Eickhoff SB, Kowalski T, de Vos K, Amunts K, Zilles K (2005) Quantitative architectectural analysis: a new approach to cortical mapping. Anat Embryol (Berl) 210:373-386.
Smith SM, Jenkinson M, Woolrich MW, Beckmann CF, Behrens TE, Johansen-Berg H, Bannister PR, De LM, Drobnjak I, Flitney DE, Niazy RK, Saunders J, Vickers J, Zhang Y, De SN, Brady JM, Matthews PM (2004) Advances in functional and structural MR image analysis and implementation as FSL. Neuroimage 23 [Suppl 1]:S208-S219.

Smith SM, Fox PT, Miller KL, Glahn DC, Fox PM, Mackay CE, Filippini N Watkins KE, Toro R, Laird AR, Beckmann CF (2009) Correspondence of the brain's functional architecture during activation and rest. Proc Natl Acad Sci U S A 106:13040-13045.

Steinmetz PN, Roy A, Fitzgerald PJ, Hsiao SS, Johnson KO, Niebur E (2000) Attention modulates synchronized neuronal firing in primate somatosensory cortex. Nature 404:187-190.

Stepniewska I, Preuss T, Kaas JH (2006) Ipsilateral cortical connections of dorsal and ventral premotor areas in new world owl monkeys. J Comp Neurol 495:691-708.

Tomassini V, Jbabdi S, Klein JC, Behrens TE, Pozzilli C, Matthews PM Rushworth MF, Johansen-Berg H (2007) Diffusion-weighted imaging tractography-based parcellation of the human lateral premotor cortex identifies dorsal and ventral subregions with anatomical and functional specializations. J Neurosci 27:10259-10269.

Toro R, Fox PT, Paus T (2008) Functional coactivation map of the human brain. Cereb Cortex 18:2553-2559.

Torquati K, Pizzella V, Della PS, Franciotti R, Babiloni C, Rossini PM, Romani GL (2002) Comparison between SI and SII responses as a function of stimulus intensity. Neuroreport 13:813-819.

Tukey JW (1994) The problem of multiple comparisons. In: The collected works of John W., vol. III Tukey (Braun HI, ed), pp. 1-300. New York: Chapman \& Hall.

Turkeltaub PE, Eden GF, Jones KM, Zeffiro TA (2002) Meta-analysis of the functional neuroanatomy of single-word reading: method and validation. Neuroimage 16:765-780.

Wasaka T, Nakata H, Akatsuka K, Kida T, Inui K, Kakigi R (2005) Differential modulation in human primary and secondary somatosensory cortices during the preparatory period of self-initiated finger movement. Eur J Neurosci 22:1239-1247.

Worsley KJ, Marrett S, Neelin P, Vandal AC, Friston KJ, Evans AC (1996) A unified statistical approach for determining significant signals in images of cerebral activation. Hum Brain Mapp 4:58-74.

Young JP, Herath P, Eickhoff S, Choi J, Grefkes C, Zilles K, Roland PE (2004) Somatotopy and attentional modulation of the human parietal and opercular regions. J Neurosci 24:5391-5399.

Zilles K, Schleicher A, Palomero-Gallagher N, Amunts K (2002) Quantitative analysis of cyto- and receptor architecture of the human brain. In Brain mapping, the methods (Mazziotta J, Toga A, eds), pp 573-602. San Diego: Academic. 